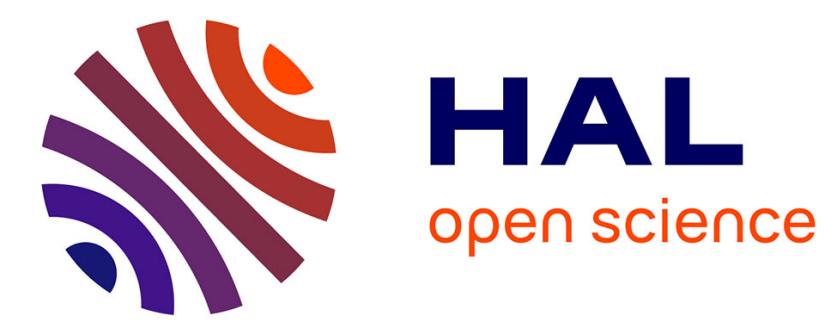

\title{
Ultrasonic vibrothermography using low-power actuators: an impact damage detection case study
}

B. Lamboul, F. Passilly, J.M. Roche, D. Balageas

\section{To cite this version:}

B. Lamboul, F. Passilly, J.M. Roche, D. Balageas. Ultrasonic vibrothermography using low-power actuators: an impact damage detection case study. 41st Annual Review of Progress in Quantitative Nondestructive Evaluation Conference (QNDE 2014), Jul 2014, BOISE, United States. hal-01132142

\section{HAL Id: hal-01132142 \\ https://hal.science/hal-01132142}

Submitted on 16 Mar 2015

HAL is a multi-disciplinary open access archive for the deposit and dissemination of scientific research documents, whether they are published or not. The documents may come from teaching and research institutions in France or abroad, or from public or private research centers.
L'archive ouverte pluridisciplinaire HAL, est destinée au dépôt et à la diffusion de documents scientifiques de niveau recherche, publiés ou non, émanant des établissements d'enseignement et de recherche français ou étrangers, des laboratoires publics ou privés. 


\section{Ultrasonic vibrothermography using low-power actuators: an impact damage detection case study}

B. Lamboul, F. Passilly, J.M. Roche, D. Balageas *

41st Annual Review of Progress in Quantitative Nondestructive Evaluation Conference (QNDE 2014) BOISE, U.S.A 20-25 juillet 2014

TP 2015-104

\section{ONERA}

THE FRENCH AEROSPACE LAB 



\title{
Ultrasonic vibrothermography using low-power actuators: an impact damage detection case study
}

\section{Vibrothermographie ultrasonore avec excitation basse énergie : application à la détection

$$
\text { d'endommagement d'impact }
$$

\author{
par \\ B. Lamboul, F. Passilly, J.M. Roche, D. Balageas * \\ * Université de Bordeaux-I2M/TREFLE, Talence, France
}

\section{Résumé traduit :}

Cet article explores le potentiel de la vibrothermographie ultrasonore, avec excitation basse énergie fournie par des disques piézoélectriques, pour la détection d'endommagement d'impact dans une plaque composite à fibres de carbone. La fréquence d'excitation est fixée dans un domaine allant de quelques dizaines à quelques centaines de $\mathrm{kHz}$ pour générer des ondes guidées dans la plaque. Les données thermiques, acquises au moyen d'une caméra moyen infrarouge, sont traitées en détection synchrone, la fréquence du signal de la porteuse étant fixée à $0,1 \mathrm{~Hz}$. L'influence des paramètres d'excitation (fréquence d'excitation, fréquence de modulation et amplitude) sur la réponse du défaut est étudiée. L'article met enfin en lumière les différents compromis à faire entre ces différents paramètres pour obtenir des cartographies de phase et d'amplitude optimales pour la détection de défauts d'impact. 



\title{
Ultrasonic vibrothermography using low-power actuators: an impact damage detection case study
}

\author{
B. Lamboul ${ }^{1 \text { a) }}$, F.Passilly ${ }^{1}$, J.-M. Roche ${ }^{1}$ and D.Balageas ${ }^{2}$ \\ ${ }^{1}$ Onera-29, avenue de la Division Leclerc- 92320 Châtillon, France. \\ ${ }^{2}$ Univ.Bordeaux-I2M, Dept. TREFLE- 33400 Talence, France. \\ ${ }^{a)}$ Corresponding author: benjamin.lamboul@onera.fr
}

\begin{abstract}
This paper investigates the use of low-power piezoelectric discs for the detection of an impact damage in a composite carbon-fibre plate using ultrasonic vibrothermography. The frequency of excitation is set in a range from tens to a few hundred $\mathrm{kHz}$ to generate guided waves in the plate. A carrier modulation signal (typically $0.1 \mathrm{~Hz}$ ) is applied to the input excitation signal to perform lock-in detection on the surface temperature signals collected by a middle-wave infrared camera. The paper studies the influence of excitation parameters (excitation frequency, modulation frequency, amplitude) on the defect response. Finally, the paper highlights some trade-offs for the detection of the considered impact damage case, based on the obtained lock-in phase and amplitude maps.
\end{abstract}

\section{INTRODUCTION}

Ultrasonic vibrothermography, also called sonothermography, or sonic IR, is a NonDestructive Testing (NDT) technique based on the local generation of heat by defects when excited by ultrasonic vibrations [1]. The mechanisms of heat production are not always readily identified, and may vary from one application to another. Heat generation is usually attributed to rubbing or clapping of defect surfaces, sometimes enhanced by viscoelastic dissipation [2,3]. In standard applications of the technique, ultrasonic excitation is achieved with a high input power (up to $2 \mathrm{~kW}$ ) sonotrode or piezoelectric stack coupled to the studied specimen with an intermediate material layer [4]. Although very effective, the coupling of high mechanical power, combined with localized heating effects at the application point are not always desirable, depending on the material of the intended NDT application. This has motivated the investigation of ultrasonic vibrothermography based on low-power actuators [5]. The comparative loss in sensitivity associated with the use of low wave amplitudes may be balanced with lock-in detection techniques and by fine tuning of the ultrasonic wavelength to optimize the defect responses. In contrast, high amplitude devices such as sonotrodes usually operate at a single resonance frequency.

Piezoelectric discs offer an interesting solution as low-power actuators for vibrothermography [5]. This type of actuator is particularly attractive from a Structural Health Monitoring (SHM) perspective since it can be permanently bonded to the structure and possibly used for different combination of in situ applicable inspection methods: guided waves purely based detection techniques [6], guided waves/ defect interaction imaging with shearography or laser vibrometry [7-10], and as in the present case, vibrothermography. The ultrasonic wavelength generated by this type of actuator is related to the diameter of the piezoelectric (the most favorable wavelength for 
wave generation is worth approximately twice the diameter [5]). Flexural $\left(\mathrm{A}_{0}\right)$ or compressive $\left(\mathrm{S}_{0}\right)$ guided waves may be generated depending on the excitation frequency. This may, at in least in theory, increase the likelihood of detection of defects, by introducing a variety in the probing mechanical solicitations. The available bandwidth around the centre wavelength furthermore makes some frequency tuning possible to optimize the defect response.

The present paper is focused on the detection of a real impact damage in a quasi-isotropic carbon/epoxy composite plate. After describing the experimental set-up, the influence of the excitation parameters (amplitude, frequency of excitation, frequency of modulation) is highlighted and a simple model of the defect response is introduced. The paper then highlights some trade-offs for optimal detection of the impact damage, based on the obtained lock-in phase and amplitude maps.

\section{EXPERIMENTAL SET-UP}

\section{Material}

The method was tested on a $4 \mathrm{~mm}$ thick, $700 \times 700 \mathrm{~mm}^{2}$ laminate, quasi-isotropic, carbon/epoxy composite plate. A $20 \mathrm{~mm}$ piezoelectric disc was bonded to the plate, $100 \mathrm{~mm}$ from the centre of an impact damage. Figure 1 shows a thermal signature of the defect obtained with flash thermography and post-processing based on the TSR (Temperature Signal Reconstruction) approach [11,12] and depth colour encoding [13]. The damage size is about 20 $\mathrm{mm}$ in diameter and presents two clear delamination depths (at 0.6 and $0.9 \mathrm{~mm}$ ), represented in yellow and green as well as some superficial small-sized delaminations. The camera used for all experiments is a middle-wave (3-5 $\mu \mathrm{m})$ FLIR camera.

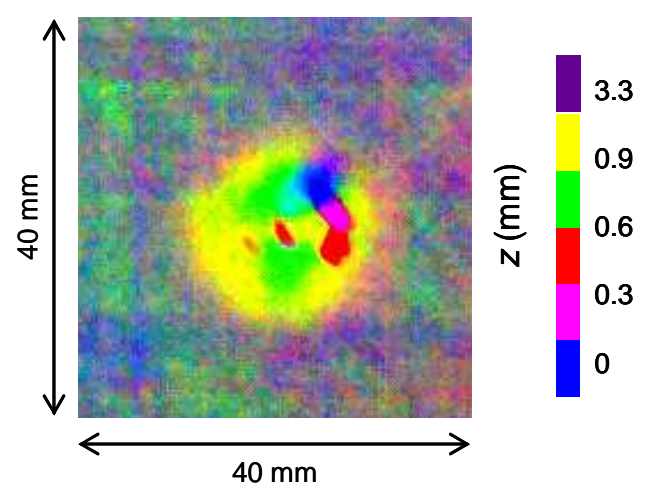

FIGURE 1. Thermal D-scan obtained on the impact damage.

\section{Signal generation for lock-in detection}

Lock-in detection with guided waves is performed using a low frequency (typically $0.1 \mathrm{~Hz}$ ) signal modulating a high frequency Continuous Wave (CW) excitation signal (tens to a few hundreds $\mathrm{kHz}$ ). The amplitude modulated (AM) signal is used, after amplification by an ENI amplifier $(50 \mathrm{~dB})$, for the excitation of the piezoelectric discs. The modulation signal is separately recorded by the camera hardware for lock-in detection. Demodulation of thermal signals was performed a posteriori by software (either MATLAB® or the FLIR lock-in detection software Thesa). 


\section{STUDY OF THE LOCAL DEFECT REPONSE}

\section{Initial selection of the excitation frequency}

The amplitude of the vibrothermographic effect generated with guided waves is highly dependent on the excitation frequency and the generated mode [5]. To avoid time-consuming frequency browsing, a linear frequency modulated (LFM) chirp, as depicted in Fig.2a, was tested for the initial selection of the operating frequency. Figure 2b) shows the average evolution of the average temperature in a defect ROI (Region Of Interest) against instantaneous frequency, for different frequency sweeps. The period of a frequency sweep is 15 s. A marked increase of the average temperature is observed in a range of frequency between $170 \mathrm{kHz}$ and $250 \mathrm{kHz}$. For the considered plate thickness $(4 \mathrm{~mm})$, the frequency range is associated with the generation of a dominant compressive $\left(\mathrm{S}_{0}\right)$ mode [5]. It is particularly interesting to note that the flexural mode (expected around $40 \mathrm{kHz}$ ) does not generate any significant vibrothermographic effect in the tested case. The remaining experiments were carried out using the lower frequency bound of the identified range of frequency, that is, $170 \mathrm{kHz}$.

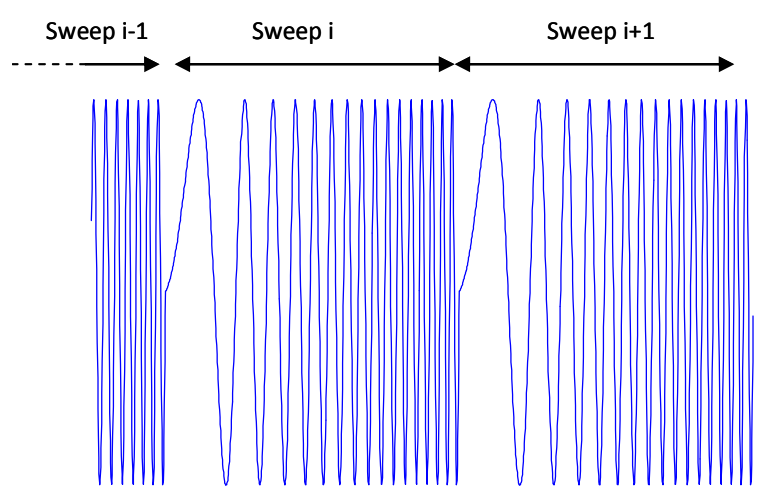

$10 \mathrm{kHz}$

\section{$300 \mathrm{kHz}$}

(a)

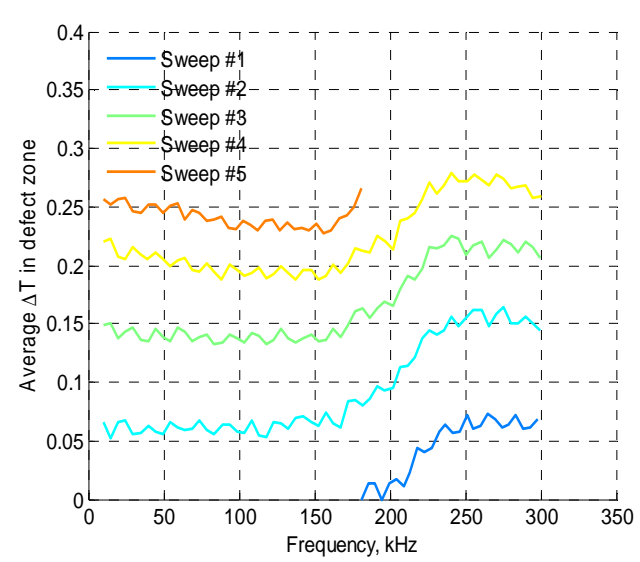

(b)

FIGURE 2. (a) LFM chirp signal used for initial frequency selection. (b) Average temperature rise in the defect region against instantaneous frequency.

\section{Signal model for the thermal response of the defect}

A simple vibrothermographic signal model is introduced to interpret the thermal response of the defect to modulated guided waves. For the sake of simplicity, all the possible heat transfers are neglected. In this case, the measured surface temperature increase reflects the uniform volumic heating of the defect region due to an equivalent vibrothermographic volumic source term. This source term is modulated as the excitation signal, taking into account a possible phase shift $\varphi$ :

$$
\rho c \frac{\partial T}{\partial t}=q_{0}\left[1+\cos \left(\omega_{\mathrm{mod}} t+\varphi\right)\right]
$$

where $\rho$ is the density, $c$ is the heat capacity of the material, $\omega_{\bmod }$ is the modulation angular frequency of the excitation signal, $\mathrm{q}_{0}$ is the amplitude of the equivalent vibrothermographic source term. Integrating (1) yields:

$$
T=\frac{q_{0}}{\rho c} t+\frac{q_{0}}{\rho c \omega_{\mathrm{mod}}} \sin \left(\omega_{\mathrm{mod}} t+\varphi\right)+T_{0},
$$

where $\mathrm{T}_{0}$ is the initial temperature. The first term corresponds to the thermal drift component of the response and the second term is the modulated amplitude part of the response. In lock-in-detection techniques, only the modulated 
part is kept for analysis, and either the amplitude information $A$ or the phase shift $\varphi$ are used to highlight regions synchronously heating up with the source term. In our simplified model $A$ is given by:

$$
A=\frac{q_{0}}{\rho c \omega_{\mathrm{mod}}} .
$$

Figure 3a highlights a typical raw signal obtained as the spatially averaged temperature in an 8x8 pixels ROI in the defect area, along with the drift signal (Fig.3b) and the modulated component (Fig.3c). Both drift and modulated components of (2) were estimated using a Least Square Minimization (LSM) identification procedure. It may be observed that the drift component significantly departs from the linear trend predicted by (2), as it could have been expected given the simplistic assumptions made in neglecting all the heat transfers. A higher order polynomial was used for the modeling of this term. In the example of Fig.3a, the image sampling rate is $2 \mathrm{~Hz}$, the modulation frequency is $0.1 \mathrm{~Hz}$, and the amplitude voltage of the excitation signal is set at $U=40 \mathrm{~V}$.

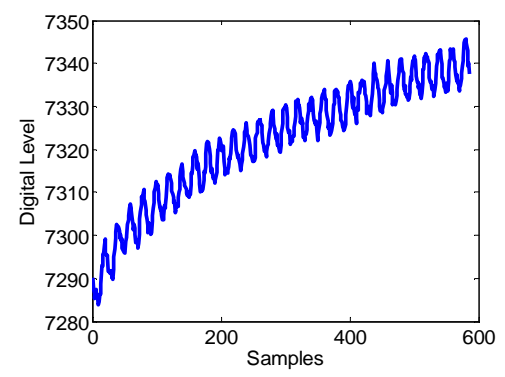

(a)

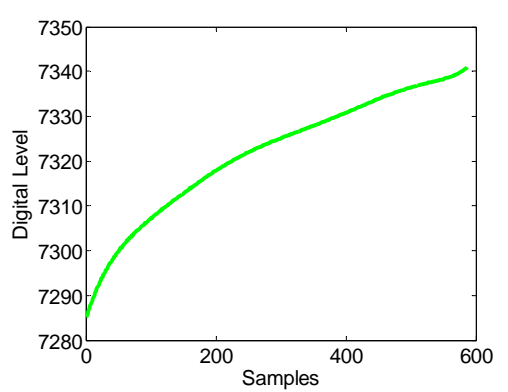

(b)

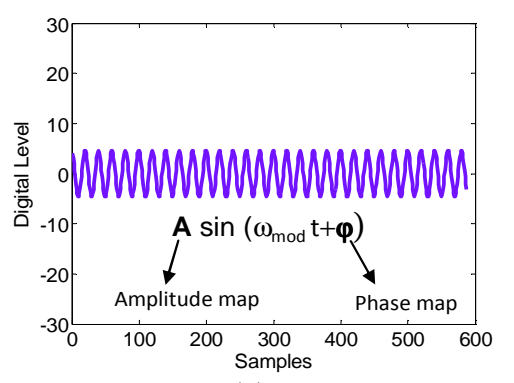

(c)

FIGURE 3. (a) Raw signal (b) Estimated drift component (c) Estimated modulated component.

\section{Influence of excitation signal amplitude and modulation frequency}

The effect of the excitation signal amplitude on the amplitude of the modulated part of the thermal signal was studied using the spatially averaged temperature over an $8 \times 8$ pixels ROI in the defect region. Figure 4 shows the obtained results when varying the excitation signal amplitude voltage $U$ applied to the piezoelectric disc from 23 to $69 \mathrm{~V}$. The estimated amplitude exhibits a linear trend with squared amplitude voltage. This is in agreement with (3) since the source term amplitude is expected to be proportional to the square of the wave amplitude and hence, to the square of the applied excitation voltage. The departure of the curve from the square trend at high voltage $(>50 \mathrm{~V})$ is mot likely due to the nonlinear behavior of the piezoelectric disc for high voltages (the generated wave amplitude is no longer proportional to the applied voltage).

The first point of the curve in Fig.4 corresponds to the minimum modulation signal that could be reliably estimated, and was obtained for $U=23 \mathrm{~V}$. The active electrical power consumed by the piezoelectric disc for this voltage is of the order of $1 \mathrm{~W}$ (taking into account the complex impedance of the piezoelectric disc at the excitation frequency of $170 \mathrm{kHz}$ ). This order of magnitude is probably a good estimation of the minimum electrical power necessary to achieve detection based on the vibrothermographic effect, with a state-of-the-art IR camera, and a 100 $\mathrm{mm}$ inspection distance. It is important to notice that, to make the most of the very low signal amplitudes at these low powers (down to +/- 1.5 camera digital levels (DL), in our case), specific attention needs to be paid to the settings of the integration time of the camera. 


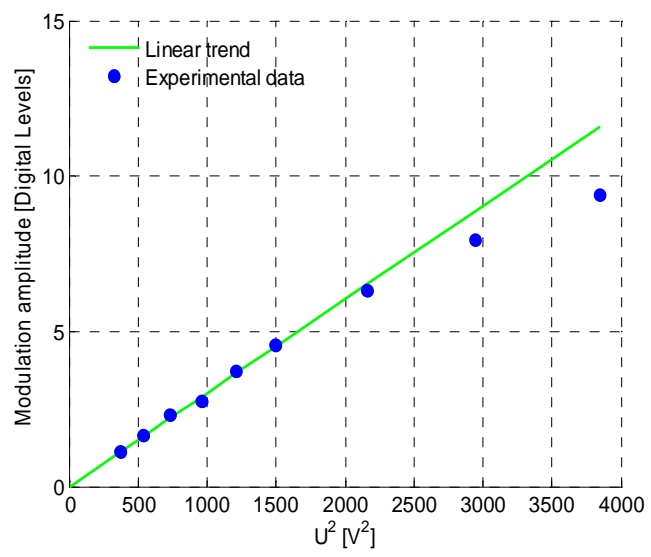

FIGURE 4. Modulation signal amplitude vs squared excitation signal voltage amplitude.

The same type of experiment was repeated while varying the modulation frequency, with a constant excitation voltage amplitude set at $U=47 \mathrm{~V}$. The graph in Fig.5 shows the obtained modulation amplitudes against the modulation period (inverse of the modulation frequency). An initial linear trend is observed for modulation periods up to a value of $5 \mathrm{~s}$, in agreement with the proposed model (3). For longer time periods, lateral heat conduction from the defect and convection probably begins to play a role and tend to lower the maximum amplitude observed in the defect region. The curve illustrates a simple trade-off for detection: larger modulation periods tend to yield higher modulation amplitudes, but for a given number of cycles of integration for lock-in detection, the larger the modulation period, the larger the acquisition duration.

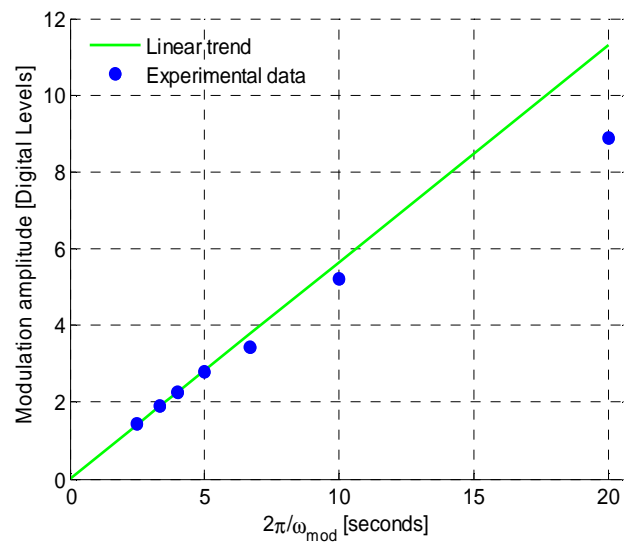

FIGURE 5. Modulation signal amplitude vs modulation period.

\section{BASIC DEFECT DETECTABILITY TRADE-OFFS}

The previous sections have emphasized the role of parameters such as the excitation amplitude and the modulation frequency to obtain large, exploitable signal modulation amplitudes in the defect region. In the perspective of applying the method to a real inspection case, it is however essential to take a look at images rather than simple signals. Moreover, phase information, which was not discussed in the previous section may also be valuable for detection. The set of images presented at Fig. 6-8 illustrates some trade-offs for the detection of the studied impact defect from amplitude and phase maps obtained with the FLIR Thesa software. The images have the same field of view, with half the piezoelectric disc on the right side of the image. 
Images of Fig. 6 were obtained with a relatively medium excitation voltage $U=46 \mathrm{~V}$, and with a 15 periods integration time at $0.1 \mathrm{~Hz}$. In spite of the short integration time used for lock-in detection, the defect is clearly visible on both maps (amplitude, Fig.6a, and phase, Fig.6b). It is however important to notice that a significant part of the image around the piezoelectric disc was saturated in Fig6.a in order to properly display the defect signature, which implies a dead zone for detection. The image dynamics is dominated in this case by the heat diffusion from the piezoelectric disc. The phase maps typically exhibit random phase jumps noise in regions where no heating occurs (either heat diffusion from the piezoelectric disc or heat generation in the defect). Overall, the defect signature contrasts quite well with this background noise. Note that in this image and all subsequent phase maps (Fig $7 \mathrm{~b}$ and $8 \mathrm{~b}$ ), the phase values were adapted for gray levels scaling to yield the best contrast. The effect of heat diffusion is also visible in Fig.6b but it is interesting to note that the phenomenon may not be so much of an issue for detection in the region close to the piezoelectric disc, contrary to the amplitude map.

The displayed in Fig7.a shows an acquisition with the amplitude voltage set to the minimum detection threshold determined in the previous section $(U=23 \mathrm{~V})$. Only a small area surrounding the piezoelectric disc is saturated in this image. Heat diffusion from the piezoelectric disc is still visible, but is far less pronounced than in Fig.6a. The amplitude level in the defect region is very low and of the same order as the surrounding thermal noise, which makes the defect barely detectable in this case. The phase map of Fig.7b provides a much better contrast for detection.

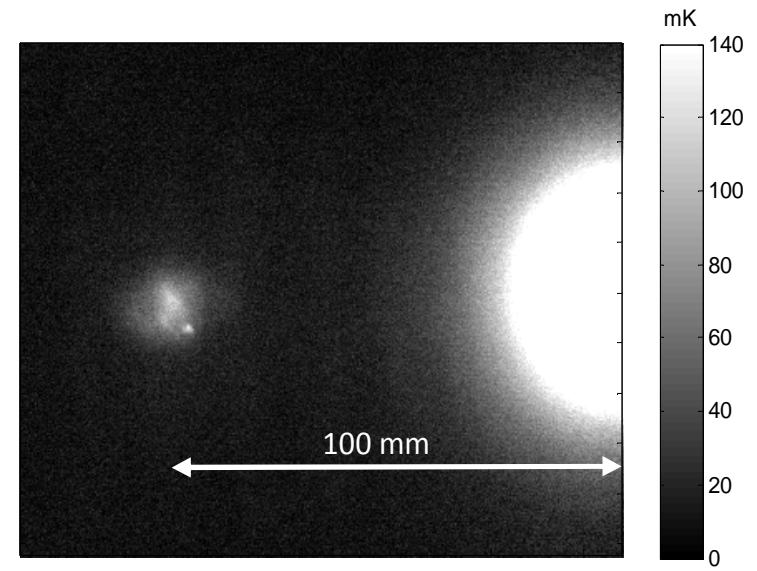

(a)

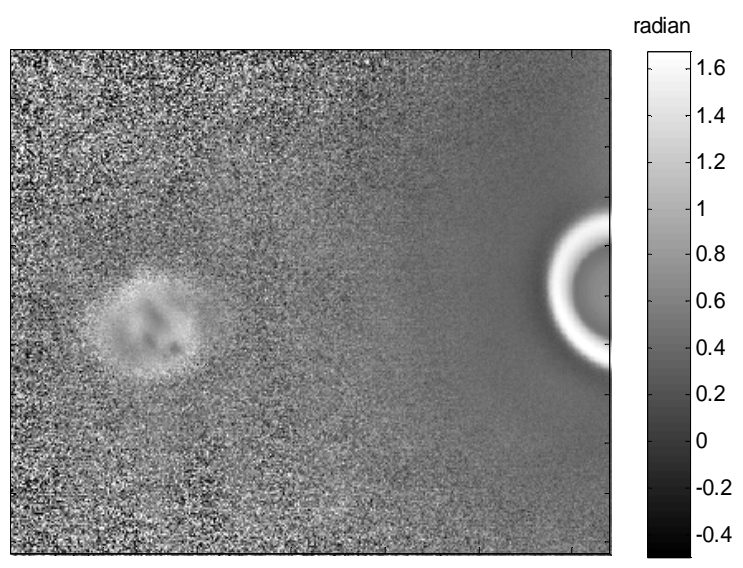

(b)

FIGURE 6. $U=46 \mathrm{~V}$, integration time: 20 periods, $10 \mathrm{~s}$ modulation period (a) amplitude map, (b) phase map.

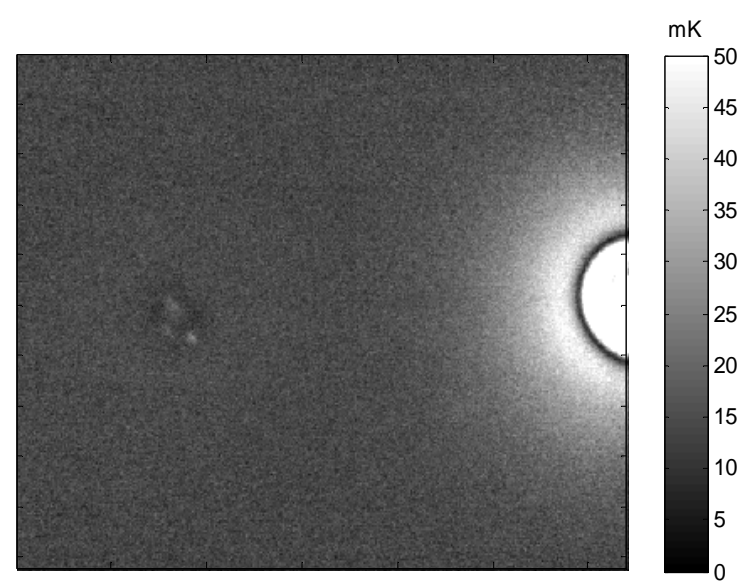

(a)

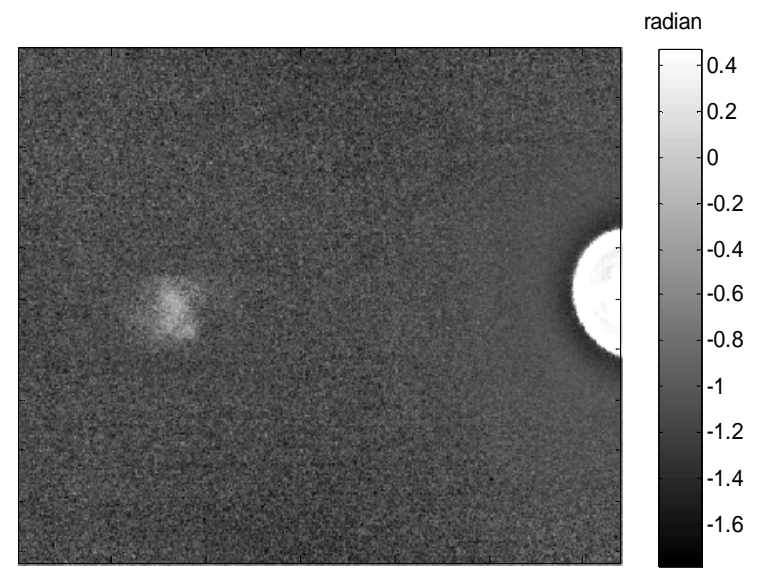

(b)

FIGURE 7. $U=23 \mathrm{~V}$, integration time: 20 periods, $10 \mathrm{~s}$ modulation period (a) amplitude map, (b) phase map. 
The final images Fig.8a and 8b show the effect of performing lock-in integration over a longer period of time, with the same configuration as used in Fig.7a-b. The defect signature in the amplitude map (Fig 8a) is largely improved and arguably provides a finer description of the defect details than the signature obtained in Fig.6a. The effect of heat diffusion is particularly reduced. This was expected, since the effect of integration over a large number of periods is that of a very narrowband filter, which selectively highlights regions with large modulated thermal signals. With purely diffusive effects, the temperature cannot be modulated far away from the piezoelectric disc (the modulated part decays exponentially as $\mathrm{e}^{-\mathrm{r} / \delta}, \delta$ being a characteristic distance depending on the plate lateral diffusivity and the modulation frequency). The counterpart of this improvement is an increased time of acquisition (by a factor of 5 compared to Fig.6b, that is, 1,000 s compared to $200 \mathrm{~s}$ ). The phase map exhibits a slightly improved signature compared to Fig.7b, but this time, the improvement is arguably not as dramatic as in the case of amplitude maps, when comparing with images obtained in Fig.6b and $7 \mathrm{~b}$.

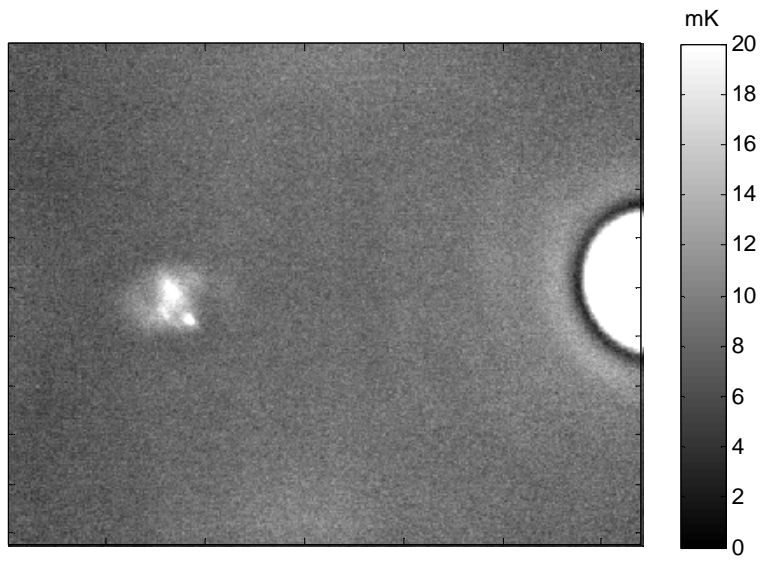

(a)

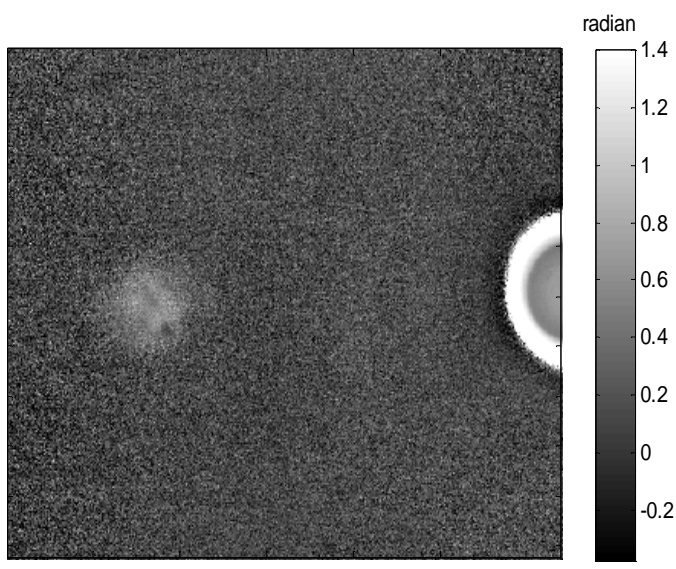

(b)

FIGURE 8. $U=23 \mathrm{~V}$, integration time: 100 periods, $10 \mathrm{~s}$ modulation period (a) amplitude map, (b) phase map.

\section{CONCLUSION}

Impact damage detection in a carbon/epoxy laminate composite plate using lock-in ultrasonic vibrothermography technique with a lower-power piezoelectric disc was investigated. The frequency of excitation was selected using a linear frequency modulated chirp signal. This approach was shown to be effective for the selection of this parameter. The frequency used in the experiments $(170 \mathrm{kHz})$ is associated with a dominant compressive $\left(\mathrm{S}_{0}\right)$ plate mode. No significant vibrothermographic effect could be obtained with the flexural mode for the tested case. The defect response was qualitatively a lot improved with an increasing excitation amplitude voltage (up to $69 \mathrm{~V}$ ). Quantitatively, the signal modulation amplitude in the defect region was observed to follow a square law trend with the excitation signal amplitude voltage used. The loss in sensitivity with the lowest excitation amplitude tested (23 V) may be partly balanced with relatively long lock-in integration durations (100 cycles). The price to pay however, is an increased time of acquisition. The same trade-off was found concerning the frequency of modulation used: low frequency provides large thermal signal in the defect area, but for a given number of cycles of integration, the acquisition is longer. Another beneficial aspect of lock-in processing over long integration time is to selectively highlight regions in the field that have their temperature modulated synchronously with the excitation source. The effect of heat diffusion around the piezoelectric disc can dominate the temperature dynamics of the investigated field and hinder defect detection. This effect was shown to be greatly reduced in amplitude images obtained with long lock-in integration times. Finally, it was noticed that phase maps provide interesting alternatives for detection, relatively independently of the integration duration. The nature of the defect signature and the contrast obtained in this case were quite different from those obtained in amplitude maps. A good detection with phase imaging seems to be achievable with only 20 cycles of integration and low excitation signal amplitude (23V). In particular, the effect 
of heat diffusion around the piezoelectric disc does not saturate the image, and might not be an impediment to detection in the region close to the piezoelectric disc.

\section{REFERENCES}

1. Mignogna, R. B., et al. "Thermographic investigation of high-power ultrasonic heating in materials." Ultrasonics 19, 159-163, (1981).

2. Reifsnider, K. L., E. Gr Henneke, and W. W. Stinchcomb, "The mechanics of vibrothermography." Mechanics of nondestructive testing. Springer US, 1980, pp. 249-276.

3. Renshaw, J., et al., "The sources of heat generation in vibrothermography." NDT \& E International 44, 736739, (2011).

4. Shepard, S. et al., "Experimental considerations in vibrothermography." Defense and Security. International Society for Optics and Photonics, 2004.

5. Krapez, J.-C., Taillade F., and Balageas D.L., "Ultrasound-lockin-thermography NDE of composite plates with low power actuators. Experimental investigation of the influence of the Lamb wave frequency." Quantitative InfraRed Thermography Journal 2, 191-206, (2005).

6. Croxford, A. J., et al., "Strategies for guided-wave structural health monitoring." Proceedings of the Royal Society A: Mathematical, Physical and Engineering Science 463, 2961-2981, (2007)

7. Osmont, D., Devillers D., and Taillade F., "Health monitoring of sandwich plates based on the analysis of the interaction of Lamb waves with damages, "SPIE's 8th Annual International Symposium on Smart Structures and Materials, International Society for Optics and Photonics, 2001.

8. Sohn, H., et al., "Automated detection of delamination and disbond from wavefield images obtained using a scanning laser vibrometer." Smart Materials and Structures 20 (2011).

9. Krapez, J.-C., et al., "Shearography: a tool for imaging Lamb waves in composites and their interaction with delaminations." Review of Progress in Quantitative Nondestructive Evaluation, Springer US, 1999, pp. 905912.

10. Lamboul, B., et al., "Impact damage detection in sandwich composite structures using Lamb waves and laser vibrometry." Review of progress Quantitative Nondestructive Evaluation, 32, AIP Publishing, 2013.

11. Shepard, S. M. "Advances in pulsed thermography" Aerospace/Defense Sensing, Simulation, and Controls. International Society for Optics and Photonics, 2001.

12. Balageas, D. L., and Roche J.-M., "Common tools for quantitative time-resolved pulse and step-heating thermography-part I: theoretical basis." Quantitative InfraRed Thermography Journal 11 (2014), pp. 43-56.

13. Roche, J. M., Leroy F. H., and Balageas D. L., "Images of TSR coefficients: a simple way for a rapid and efficient detection of defects." Materials Evaluation 72, 73-82, (2014) 



\section{ONERA}

BP 72 - 29 avenue de la Division Leclerc - 92322 CHATILLON CEDEX - Tél. : +33 146734040 - Fax : +33 146734141

w w w. onera.f r 